\title{
Experiência de ampliação de escuta com utilização da ferramenta PRACTICE para atendimento voluntário de paciente com dor orofacial crônica
}

\author{
Expansion of listening experience with use of the PRACTICE tool for \\ voluntary assistance of patients with chronic orofacial pain
}

\author{
Helen Conceição Moreira Camargos', Karina Viana Brandão', Luiz Paulo Nunes Ferreira Tomaz', \\ Ariovaldo Alberto da Silva Júnior ${ }^{2}$, Paulo Henrique Faleiro dos Santos ${ }^{3}$ \\ 'Discente do $5^{\circ}$ ano do curso de Medicina na Universidade José do Rosário Vellano \\ ${ }^{2}$ Docente, Doutor em Neurologia pela Universidade Federal de Minas Gerais - UFMG \\ ${ }^{3}$ Psicólogo, Mestre em Psicologia pela Universidade Federal de Minas Gerais - UFMG
}

\begin{abstract}
Camargos HC, Brandão KV, Tomaz LP, Silva Júnior AA, Santos PH. Experiência de ampliação de escuta com utilização da ferramenta PRACTICE para atendimento voluntário de paciente com dor orofacial crônica.
\end{abstract}

Headache Medicine. 2013;4(4):85-90

\section{RESUMO}

Justificativa e Objetivos: A dor crônica afeta aproximadamente $30 \%$ da população mundial e exige do médico considerável perspicácia para efetivar um diagnóstico que permita estabelecer relações entre as expressões dolorosas e o que esteja por trás delas. Este trabalho objetiva descrever a experiência prática de um grupo de estudantes de medicina, no exercício da ampliação da escuta de pacientes com dor crônica. Método: Relato de experiência de estudantes de medicina que foram inseridos numa equipe multidisciplinar e atenderam, como voluntários, durante mais de dois anos, pacientes com dor crônica, utilizando como roteiro das entrevistas a ferramenta PRACTICE. Resultados: A ferramenta PRACTICE se mostrou útil para direcionar a anamnese, que demandou maior tempo quando comparada a uma anamnese sem sua utilização. Os estudantes se consideraram mais preparados para abordar paciente com dor crônica em sua futura prática profissional. Conclusão: A ampliação da escuta deve ser valorizada no atendimento do paciente com dor crônica e deve ser estimulada ainda no período de formação médica. A ferramenta PRACTICE pode auxiliar a ampliação da escuta de paciente com dor crônica.

Palavras-chave: Dor crônica; Ferramenta PRACTICE; Ampliação da escuta

\section{RESUMO}

Background and Objectives: Chronic pain affects approximately $30 \%$ of the world population and requires considerable insight to the doctor to confirm a diagnosis which would establish relations between expressions painful and what is behind them. This paper aims to describe the experience of a group of medical students, in enlargement exercise of listening to patients with chronic pain. Method: Experience report of medical students who were entered in a multidisciplinary team and met as volunteers for more than two years, patients with chronic pain, using series of interviews as the tool PRACTICE. Results: PRACTICE proved useful tool to guide the interview, which required more time when compared to an interview without their use. Students felt more prepared to address patient with chronic pain in their future professional practice. Conclusion: The expansion of listening should be considered for treatment of patients with chronic pain and should be encouraged even in the period of medical training. PRACTICE The tool can assist the expansion of listening to patients with chronic pain.

Keywords: Chronic pain; PRACTICE tool; Expansion of listening 


\section{INTRODUÇÃO E JUSTIFICATIVA}

Para a Associação Internacional de Estudo da Dor (IASP), (1) a dor se constitui como uma experiência emocional e sensitiva desagradável, associada com uma lesão tecidual real ou potencial, ou descrita em termos de tal lesão.

A dor, enquanto sintoma ou até mesmo um sinal, pode ter diversas causas, o que exige do médico considerável perspicácia para efetivar um bom diagnóstico, ou seja, um diagnóstico que permita estabelecer relações entre as expressões dolorosas e o que esteja por trás delas. ${ }^{(2)}$

Estima-se que a dor crônica acometa aproximadamente $30 \%$ da população mundial, no entanto, sua epidemiologia ainda é pouco descrita no Brasil. (3) Desta forma, estudiosos do assunto já a consideram um problema de saúde pública e ensejam maior dedicação ao seu estudo. ${ }^{(4)}$

Observa-se que alguns pacientes com dor crônica, mesmo adequadamente tratados, com terapia farmacológica, não apresentam melhora ou mesmo a apresentam de forma bastante modesta e que estes, em sua maioria, apresentam sinais e sintomas que lhes conferem uma demanda de caráter psicossocial. Diante desta constatação, um grupo de estudantes de medicina, da Universidade José do Rosário Vellano (Unifenas-Belo Horizonte), sob a orientação do professor neurologista Dr. Ariovaldo da Silva Júnior, decidiram lançar mão da pesquisa científica no intuito de compreender o mister que envolve tal fato, até então pouco abordado na literatura médica, embora já relatado em outras áreas da saúde.

Sabe-se que o subjetivo, o impalpável, só pode ser compreendido se soubermos ouvir não apenas no sentido estrito da palavra, mas ouvir dando significados ao que se ouve e ao que se vê, pois, é dito que "o corpo fala", talvez até mais do que é expresso verbalmente.

"Se o corpo não falasse a palavra não teria sentido. Seria como a fala de um robô, absolutamente sem expressão."

Gaiarsa ${ }^{(5)}$

Para Neubern ${ }^{(2)}$ o corpo é assemelhado, pelo médico, a um laboratório, onde as influências indesejáveis e subjetivas, capazes de contaminar a validade do saber médico, são afastadas ou pretensamente controladas. Corroborando com o autor Stengers, ${ }^{(6)}$ que afirmou que a fala, a emoção, a história e o símbolo ligados à dor são convertidos (pelo médico) em sinais secundários ou apenas ignorados em sua complexidade.

Prova da dificuldade da classe médica em saber ouvir o paciente são os dados de um estudo recente, publicado no The Journal of the American Medical Association, ${ }^{(7)}$ constatando que $72 \%$ dos médicos americanos interrompem a fala do paciente depois de apenas 23 segundos, em média.

Cientes da responsabilidade dos profissionais da saúde em abordar adequadamente o paciente portador de dor crônica, este trabalho propõe-se a descrever a experiência de um grupo de estudantes de medicina, inseridos num contexto multidisciplinar, exercitando a ampliação da escuta direcionada pela ferramenta PRACTICE.

\section{$\overline{\text { MÉTODO }}$}

\section{Estruturação e dinâmica do Projeto AmbDof}

De março de 2010 a julho de 2012, uma equipe multidisciplinar constituída por neurologista, dentistas, psicólogo, assistente social e acadêmicos de medicina atenderam, voluntariamente, em ambulatórios, pacientes com algum tipo de dor crônica, em sua maioria portadores de dor orofacial, formando assim um ambulatório de estudo da dor (AmbDof). Os atendimentos foram realizados por meio de encontros mensais no Centro de Especialização e Treinamento da Odontologia (CetroUnidade Belo Horizonte, Belo Horizonte/MG).

Inicialmente foi realizada entrevista semiestruturada que investigava semiologicamente o quadro doloroso do paciente, bem como a presença de hábitos parafuncionais, como apertamento mandibular, bruxismo, entre outros. Posteriormente, dentistas realizaram a palpação muscular pericraniana e cervical destes pacientes, procurando pontos álgicos (trigger points). Prosseguia-se com avaliação neurológica, por meio da qual eram adequados os diagnósticos segundo os critérios da ICDH-2004. Após esta avaliação multiprofissional, a equipe selecionava casos nos quais percebiam maior dificuldade de abordagem ou mesmo sinais de demanda psicossocial, encaminhando-os para uma avaliação mais ampla, realizada por acadêmicos de medicina, que efetivaram a escuta destes pacientes fazendo uso da ferramenta PRACTICE (descrita a seguir), sob a supervisão de psicólogo e/ou uma assistente social com formação em psicoterapia. 


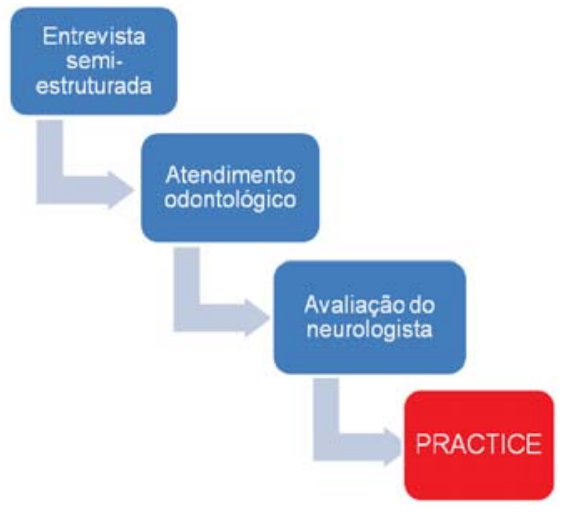

\section{A ferramenta PRACTICE}

O PRACTICE8 é um instrumento que funciona como um roteiro para obtenção de informações, focado na resolução de problemas. Cada letra significa uma área de investigação. Todos os acadêmicos envolvidos no Projeto AmbDof foram previamente familiarizados com a ferramenta e treinados para sua utilização como instrumento direcionador da anamnese.

O PRACTICE, portanto, nada mais é senão um mnemônico cujas letras, derivadas do inglês, significam:

\begin{tabular}{|l|l|l|}
\hline Abreviações & Inglês (Original) & Português (Tradução) \\
\hline P & Presenting problem & Problema apresentado \\
\hline R & Roles and structure & Papéis e estrutura \\
\hline A & Affect & Afeto \\
\hline C & Communication & Comunicação \\
\hline T & Time in life cycle & Tempo no ciclo de vida \\
\hline I & Illness in Family & Doenças na Familia \\
\hline C & Coping with stress & Enfrentando o estresse \\
\hline E & Ecology & Ecologia \\
\hline
\end{tabular}

Gusso et al. ${ }^{(8)}$

Para Gusso et al. ${ }^{(8)}$ o PRACTICE geralmente é usado em conferências familiares, porém, no contexto deste estudo, a ferramenta foi utilizada como direcionador na entrevista de pacientes, que, em sua maioria, participavam da consulta desacompanhados. Desta forma abordou-se em cada atendimento:

Problema: Como o paciente percebe, define e enfrenta o problema atual (por exemplo, sua dor).

Papéis e estrutura: Aprofunda aspectos do desempenho dos papéis de cada um dos familiares e como eles evolvem a partir dos seus posicionamentos.

Afeto: Como se estabelecem as trocas de afeto entre os membros e como esta troca reflete e interfere no problema apresentado.
Comunicação: Como acontecem as diversas formas de comunicação entre as pessoas.

Tempo no ciclo de vida: Correlaciona o problema com as dificuldades e as tarefas esperadas dentro das diversas fases do ciclo de vida.

Doenças na família (passadas e presentes): Resgata a morbidade familiar e o modo de enfrentamento nas situações pregressas. Trabalha com a longitudinalidade do cuidado e a importância do suporte familiar.

Enfrentando o estresse: Como a pessoa/família lida com o estresse. A equipe parte das experiências anteriores e analisa a atual. Identifica fontes de recursos internos, explora alternativas de enfrentamento se requeridas e interfere se a crise estiver fora de controle.

Ecologia (ou meio ambiente): Identifica o tipo de sustentação familiar e como podem ser usados os recursos disponíveis. Representada por meio do instrumento "ecomapa", um diagrama das relações entre a família e a comunidade e ajuda a avaliar os apoios e suportes disponíveis e sua utilização pela família.

Deste modo, o PRACTICE objetivou direcionar a ampliação da escuta dos pacientes, o que foi facilitado pelos profissionais da área psicossocial que oportunamente interviam na entrevista, a fim de pontuar situações nas quais percebiam necessidade de maior esclarecimento e aprofundamento.

\section{Critérios utilizados para finalizar a experiência}

Após cada dia de atendimento, os alunos, conjuntamente com o psicólogo e com a assistente social, faziam discussões crítico-reflexivas acerca dos casos atendidos, pontuando as visões de cada um sobre o caso, bem como as dificuldades encontradas na exploração da(s) queixa(s). Muitas vezes, os alunos foram também submetidos à escuta ampliada, no contexto de trazer à tona suas questões pessoais que se manifestavam direta ou indiretamente em sua condução das anamneses e/ou em suas reações frente aos conteúdos apresentados pelos pacientes.

Com o decorrer do tempo, visto que a experiência adquirida pelos alunos já os deixavam confortáveis o suficiente para abordar o paciente de maneira mais integral, os profissionais envolvidos e os estudantes, em comum acordo, decidiram finalizar o período de experiência clínica e compartilhá-la com a comunidade científica. Ao refletir sobre a riqueza do aprendizado adquirido, pensou-se na melhor maneira de transmiti-lo, a fim de incentivar ações semelhantes. 


\section{Delineamento do tipo de estudo para divulgação dos resultados}

A natureza deste objeto impôs a adoção dos preceitos metodológicos do estudo qualitativo que, de acordo com Pope \& Mays, ${ }^{(9)}$ está relacionado aos significados que as pessoas atribuem às experiências do mundo social e como o compreendem. Tenta interpretar os fenômenos sociais em termos dos sentidos que as pessoas thes dão.

Diferente da pesquisa quantitativa, os métodos qualitativos consideram a comunicação do pesquisador em campo como parte explícita da produção de conhecimento, em vez de simplesmente encará-la com uma variável a interferir no processo. A subjetividade do pesquisador, bem como dos sujeitos do estudo, tornamse parte do processo de pesquisa (Flick). ${ }^{(10)}$ Além disso, as pesquisas qualitativas fazem emergir aspectos subjetivos e atingem motivações não explícitas, ou mesmo conscientes, de maneira espontânea. Estes aspectos nos levaram a usar como instrumento de coleta de dados a entrevista semiestruturada focalizada, uma vez que ela permite obter dados, opiniões sobre tema bem definido e explicitamente delimitado.(11) No total foram quatro estudantes que permaneceram até o final do Projeto e concordaram em relatar suas experiências, procedendo a assinatura do Termo de Consentimento Livre e Esclarecido (TCLE), de acordo com as determinações da Resolução 196/96 do Conselho Nacional de Saúde (Brasil, 1996), a qual discorre sobre o respeito devido à dignidade humana. Neste trabalho, fez-se cumprir ainda o direito do anonimato, omitindo-se os nomes dos entrevistados e foram descritos apenas seus relatos.

Os dados das entrevistas foram organizados de acordo com os pressupostos de pesquisa qualitativa apontados por Minayo ${ }^{(12)}$ : leituras e releituras de cada entrevista a fim familiarizar com o conteúdo expresso e, assim, estabelecer uma compreensão dos dados coletados, confirmando ou não os pressupostos da pesquisa e/ou responder às questões formuladas e ampliar o conhecimento sobre o assunto pesquisado. Procedeu-se então o pinçamento das unidades de registro, relendo-as quantas vezes se fizeram necessárias no intuito de apreender o sentido latente e expresso nelas para uni-las por convergência e formar as unidades temáticas de análise.

\section{RESULTADOS E DISCUSSÃO}

A entrevista com os acadêmicos, que participaram do projeto AmDof, constou das seguintes questões:
1) Descreva como foi a sua experiência de atendimento aos pacientes no ambulatório do CETRO. O que mais the chamou atenção nesse processo?

2) $\bigcirc$ que é ampliação de escuta para você?

3) Sua experiência no atendimento ao paciente com dor crônica, utilizando o PRACTICE, foi diferente em algum aspecto quando comparado à sua anamnese em outros ambulatórios nos quais não a utilizou? Se sim, em que diferiu?

4) Você acredita que esta prática da ampliação da escuta auxiliará em sua futura prática profissional? Se sim, como?

A análise compreensiva permitiu localizar na fala dos entrevistados as unidades de registro que Minayo ${ }^{(12)}$ diz referir-se aos elementos obtidos através de decomposição do conjunto da mensagem.

As unidades de registro foram obtidas por meio do pinçamento central das respostas de cada pergunta e foram agrupadas de acordo com a similaridade de seus conteúdos, que receberam um título abrangente para caracterizar a ideia geral das respostas.

Os estudantes demonstraram, por meio de suas falas, que a experiência de atendimento aos pacientes no ambulatório de dor foi positiva, o que lhes permitiu ampliar a escuta, compreender o paciente em sua totalidade e os ajudaram a criar uma visão crítica sobre a anamnese tradicional:

1) Aprender a ouvir o outro:

"Foi uma experiência ímpar, na qual pude dedicar meu tempo para ouvir, mas ouvir de verdade."

"Chamou-me atenção o quanto que as pessoas precisam falar, expressar seus sentimentos, e como que somos carentes do ouvir em nossa sociedade."

2) Compreender o paciente em sua totalidade:

"Me permitiu ver o paciente como um ser humano único, fragilizado por sua dor, inserido em um contexto biopsicossocial, e com uma história de vida impregnada de valores culturais, espirituais e sociais."

"Acho que o que mais me chamou atenção foi como cada um de nós enfrenta ou simplesmente vive e convive com a dor de maneiras diferentes."

3) Questionar a anamnese tradicional

"O que mais me chamou atenção foi a falta de sensibilidade e a estruturação desnecessária que durante a formação médica adquirimos."

Sabe-se que a medicina foi fundamentada no estudo dos componentes biológicos do corpo para 
construir suas teorias, elaborar seus diagnósticos e determinar seus tratamentos. ${ }^{(13)}$ Assim, o século XIX foi marcado por um processo de rápido desenvolvimento do saber, no qual houve uma supervalorização do método científico. Isso, na medicina, implicou em incontestáveis avanços, mas trouxe consigo certo esquecimento de seu lado humanístico que agora se faz ressurgir. Para Cassel, (14) a tarefa da medicina no século XXI será a descoberta da pessoa, descobrindo as origens da doença e do sofrimento e com este conhecimento desenvolver métodos para o alívio da dor, e, ao mesmo tempo, revelar o poder da própria pessoa.

Ao serem questionados quanto ao conceito de ampliação de escuta, os estudantes a definiram como: saber ouvir em profundidade e considerar os aspectos biopsicossociais do paciente.

1) Ouvir em profundidade

"É ir além do que o paciente nos conta. É ouvir e saber explorar em profundidade o que é contado."

"Enxergar através das entrelinhas do discurso verbal e não verbal do paciente."

2) Considerar aspectos biopsicossociais do paciente

"É ter uma visão holística, não apenas ouvir a queixa física do paciente e sim conhecê-lo melhor e ver que por trás de um sintoma há uma realidade psíquica e espiritual individualizada, que interage com seu meio ambiente, sociedade e cultura da sociedade na qual está inserido."

"É conseguir atender o paciente na sua integralidade, observando as peculiaridades individuais de cada um."

De modo geral, os estudantes consideraram a ferramenta PRACTICE como útil e facilitadora do processo de ampliação da escuta, embora aumente o tempo da consulta:

"Com o PRACTICE ao alcance dos olhos durante a anamnese eu me sentia mais segura e não esquecia de abordar os aspectos biopsicossociais do paciente, o que nem sempre fazemos numa consulta comum."

"Observei que o tempo de atendimento em uma consulta, utilizando o PRACTICE, é maior, porém o paciente se sente acolhido e acredita mais no tratamento."

"A ferramenta PRACTICE em minha opinião possibilita conectar informações que poderiam passar despercebidas, contribuindo para o diagnóstico e tratamento. Utilizá-lo é de suma importância para garantir qualidade de vida e em última análise ter a sensibilidade de intervir no fator complicador e perpetuador da referida dor."
"Aprofundamos nos aspectos psicossociais em detrimento dos aspectos puramente biológicos, tentando ao fim correlacioná-los."

Os acadêmicos que participaram do projeto consideraram que a experiência da ampliação da escuta será um fator diferencial em suas formações e que certamente os auxiliará na futura prática profissional e apontaram como aspectos positivos: maior segurança na abordagem do paciente, redução do número de consultas, diferenciação no mercado de trabalho e facilitação do diagnóstico etiológico da dor. Exemplos:

1) Maior segurança na abordagem do paciente:

"Sinto-me bem mais segura em abordar o paciente e sempre reservo um tempinho da anamnese para explorar estas questões de como o paciente lida com seu problema, tento encontrar coincidências entre suas queixas e sua história de vida, seus problemas pessoais...(...) o vínculo que formamos é muito maior."

2) Redução do número de consultas

"Ao desprender um tempo maior na consulta, permite compreender o contexto em que o paciente está inserido, podendo desta forma realizar a melhor conduta, evitando assim que o paciente retorne várias vezes ao atendimento."

3) Diferencial no mercado de trabalho

"A familiarização com a ampliação de escuta será com certeza um diferencial importante para o atendimento clínico profissional. (...) tendo em mente, que a medicina também é uma arte, saber conectar, a sensibilidade da escuta atenta ao paciente (...); fica claro que ter participado do projeto contribuiu de sobremaneira para formação de uma opinião sólida sobre a necessidade de médicos que tenham este perfil, iá que atualmente são escassos."

4) Facilitação do diagnóstico etiológico da dor

"Se aprendermos a deixar o paciente falar, ele nos dará toda a informação necessária, seja por meio do discurso ou por linguagem corporal. Uma vez feito isso, saberemos o que perguntar e identificaremos de forma mais fácil o "gatilho" da dor do paciente e não apenas tratar a consequência da mesma, mas sim a causa."

A integralidade é um dos princípios doutrinários do Sistema Único de Saúde (SUS), instituído pela Constituição desde 1988. E embora, desde então, o Brasil tenha se esforçado por fazê-lo cumprir, ainda há muito que se fazer para que a pessoa doente não seja vista sob a ótica reducionista das subespecialidades. Para se alcançar uma visão completa do sujeito adoecido e assim, 
compreender a causa dessa(s) doença(s) o médico necessita de habilidades de comunicação para fazer uma leitura global do contexto daquela doença, considerando o que o paciente expressa, verbalmente ou não.

Sobre isso, Helman (1994) apud Caprara \& Franco ${ }^{(15)}$ trazem que, em se tratando dos padrões de comunicação verbal e não verbal, assim como a variedade de padrões comunicacionais, são muitos os problemas que surgem na relação médico-paciente destacando-se: a dificuldade de compreensão, por parte do médico, das palavras utilizadas pelo paciente para expressar a dor, o sofrimento; a falta ou a dificuldade de transmitir informações adequadas ao paciente; a dificuldade do paciente na adesão ao tratamento. Desta forma, o PRACTICE se mostra como um facilitador do processo de aprofundamento da escuta, mas não exclui a necessidade de um treinamento do profissional para a captação da linguagem corporal do paciente, o que permitirá uma melhor compreensão do problema apresentado.

\section{CONCLUSÃO}

Percebe-se surgir um novo cenário da medicina, no qual o paciente é reconhecido como principal instrumento de cura, exercendo papel fundamental no sucesso do seu próprio tratamento. Desta forma, vê-se a construção de uma nova relação médico-paciente, no qual o médico se colocará cada vez mais como facilitador ou orientador do processo de autocura, ombreando com o paciente a responsabilidade de buscar sempre a origem e o tratamento para sua doença. Assim sendo, saber ouvir e interpretar sua linguagem verbal e corporal, bem como compreender o contexto social, cultural e ambiental no qual se insere o sujeito adoecido, se faz de suma importância para se exercer a medicina do século XXI.

A integralidade do cuidado parece ser viabilizada pelo atendimento conjunto multiprofissional. A ferramenta PRACTICE somada à orientação de profissionais da área psicossocial se mostrou como facilitadora do processo de ampliação da escuta.

Diante de tais fatos, conclui-se que a escuta ampliada deve ser valorizada no atendimento do paciente com dor crônica e certamente também naqueles com outras afecções à saúde. Sua estimulação, ainda no período de formação médica, se mostra um diferencial importante, que contribui para maior segurança e humanização no atendimento, interferindo positivamente na relação médico-paciente e facilitando assim o cuidado integral ao sujeito adoecido.

\section{REFERÊNCIAS}

1. International Association for the Study of Pain - IASP. Part III: Pain Terms, A Current List with Definitions and Notes on Usage. 2ed. Copyright (C) 2013. Disponível em: < http://www.iasp-pain.org/ Content/NavigationMenu/GeneralResourceLinks/ PainDefinitions/default.htm>. Acesso em 15 de Março de 2013.

2. Neubern MS. Hipnose, dor e subjetividade: considerações teóricas e clínicas. Psicologia em Estudo. 2009; 14(2):303-10.

3. Anderson C, Almeida DB, Vall J. Perfil do paciente com dor crônica atendido em um ambulatório de dor de uma grande cidade do sul do Brasil. Rev Dor 2011 ;12(4). Disponível em $<$ http://www.scielo.br/scielo.php?script=sci_arttext\&pid=S1806$00132011000400003 \&$ lng $=$ en\&nrm $=i$ iso $>$. Acesso em 30 de março 2013. http://dx.doi.org/10.1590/S180600132011000400003.

4. Holtz W, Stechman J. Epidemiologia da dor em pacientes de Curitiba e região metropolitana. Rev Dor. 2008;9(2): 1217-24.

5. Gaiarsa JA. O corpo fala? Motriz. 2002;8(3):85-90.

6. Stengers I. Le médecin et le charlatan. In Neubern MS. Hipnose, dor e subjetividade: considerações teóricas e clínicas. Psicologia em Estudo. 2009; 14(2), p. 303-10.

7. Marvel MK, Epstein RM, Flowers K, Beckman HB. Soliciting the patient's agenda: have we improved? JAMA. 1999; 281 (3): 283-7.

8. Gusso G, Lopes JMC, et al. Tratado de Medicina de Família e Comunidade - Princípios, Formação e Prática. Artmed, Vol. I, 2012.

9. Pope C, Mays N. Pesquisa Qualitativa na Atenção à Saúde. 2a. ed. Porto Alegre: Artmed, 2005.

10. Flick U. Introdução à pesquisa qualitativa. 3a. ed. Porto Alegre: Artmed, 405p, 2009.

11. Lima MC. Monografia: a engenharia da produção acadêmica. São Paulo: Saraiva, 2004.

12. Minayo MCS. (Org.). (2001). Pesquisa social: teoria, método e criatividade. Rio de Janeiro: Vozes.

13. Gallian DMC. A (re)humanização da medicina. Centro de História e Filosofia das Ciências da Saúde da Unifesp-EPM. Volume 34, número 4, 2001-2002. Disponível em: < http://www.unifesp.br/ dpsiq/polbr/ppm/especial02a.htm>. Acesso em 07 de Abril de 2013.

14. Cassel $E$. The nature of suffering and the goals of medicine. Oxford: Oxford University Press, 1991.

15. Caprara A, Franco ALS. A relação paciente-médico: para uma humanização da prática médica. Cad Saúde Pública 1999; 15:647-54

$\overline{\text { Correspondência }}$
Helen Conceição Moreira Camargo
Núcleo de Pesquisa UNIFENAS
Rua Líbano, 66 - Bairro Itapoã
31710-030-Belo Horizonte, MG

Received: December 2, 2013

Accepted: December 20, 2013

Headache Medicine, v.4, n.4, p.85-90, Oct./Nov./Dec. 2013 